\title{
The hunt for Julia sets with positive measure
}

\begin{abstract}
Arnaud Chéritat
To appear in Complex Dynamics, Families and Friends, Editor Dierk Schleicher.

Abstract. This article is based on the talk given by the author for the conference. We will explain the status that had reached, during Hubbard's 60th birthday conference, Douady's plan to produce Julia sets with positive Lebesgue measure. We will also quickly mention the progress done since then.
\end{abstract}

\section{Introduction}

The title of the talk I gave at Hubbard's 60th birthday conference was "Are there Julia sets with positive measure?". Four months later I gave a talk in Denmark where the first two words were inverted and the question mark became a period.

I do not know when Adrien Douady set up his plan for finding a Julia set with positive measure, but in 1998 he offered me to study it for my $\mathrm{PhD}$ thesis. He added that his opinion about whether or not such Julia sets existed depended on his mood. If the plan worked it would solve an open conjecture. But in case of failure, it would not say anything on the measure of the objects he was looking at (quadratic Julia sets with a Cremer point). ${ }^{1}$

When I finished my thesis in 2001, I had convinced Adrien that his plan would work, by proving it would follow from a pair of reasonable conjectures, supported by analogy with known results for Julia sets with a bounded type Siegel disk, also supported by computer pictures.

Adrien told me cylinder renormalization could help in proving these conjectures and had me and Xavier Buff meet and begin a very productive collaboration on quadratic Julia sets. I also went to Toronto for six months where I met Yampolsky and had many discussions with him. However cylinder renormalization turned out to be difficult. We lacked first an invariant class, second an argument to turn this into a proof of my conjectures.

\footnotetext{
${ }^{1}$ We now have examples with positive measure, but it is still unknown whether or not some of them have measure 0 .
}

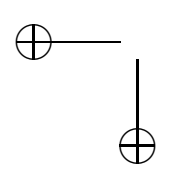


An invariant class was found by Inou and Shishikura in 2003.

To turn it into a proof still required some time and effort. I remember three key periods. First, I was asked to write a review for the French Mathematical Society on the book "The Mandelbrot Set, Theme and Variations" edited by Tan Lei [T]. In the process, I learnt important methods in holomorphic dynamics, and this knowledge proved very useful. Second, this is in the favorable atmosphere of Hubbard's 60th birthday conference that I saw the end of the tunnel, i.e. how things may fit together to provide a proof. The third period was when Xavier and I worked hard during the following summer holiday to make it work.

\section{Crash-course}

This section introduces some terminology for the non-experts or readers in the distant future. See for instance [M] for a more thorough introduction.

In this article, it is understood that we work with complex polynomials, i.e. with some $P \in \mathbb{C}[X]$. A quadratic polynomial is just any degree 2 polynomial. We are interested in the iteration of $P$, i.e. in the behavior of sequences $z_{n}$ defined by $z_{n+1}=P\left(z_{n}\right)$, and how this behavior depends on $z_{0}$ and on $P$. Here $P^{n}$ will refer to the composition $P \circ \cdots \circ P$, and not to the product.

The orbit of a point is the set $\left\{P^{n}(z) \mid n \in \mathbb{N}\right\}$. A periodic point is a point $z_{0}$ such that $z_{k}=z_{0}$ for some $k>0$. Its period is the least value of $k$ such that this hold. A cycle is the orbit of a periodic point. A period one periodic point is also called a fixed point. Its multiplier is the derivative $\lambda=\left(P^{k}\right)^{\prime}\left(z_{0}\right)$. The periodic point is repelling if $|\lambda|>1$, indifferent if $|\lambda|=1$, attracting if $|\lambda|<1$ and superattracting if $\lambda=0$. Superattracting is considered as a subcase of attracting. For instance, $\infty$ is a superattracting fixed point of any polynomial of degree $>1$.

The periodic point $z_{0}$ is linearizable when there exists a homeomorphism $\phi$ from a neighborhood of $z_{0}$ to a neighborhood of 0 such that $\phi \circ P^{k} \circ$ $\phi^{-1}(z)=\lambda z$ holds in a neighborhood of $z_{0}$. For dimension one analytic maps, the existence of a continuous conjugacy to the linear part is known to imply the existence of an analytic one.

If $|\lambda| \neq 0$ and $|\lambda| \neq 1$, then the periodic point is automatically linearizable. If $|\lambda|=0$ then it can be conjugated to $z \mapsto z^{d}$ in a neighborhood of 0 , for some integer $d \geq 2$. If $|\lambda|=1$, then write $\lambda=e^{2 i \pi \theta}$ for some $\theta \in \mathbb{R}$. This $\theta$ is called the rotation number. If $\theta \in \mathbb{Q}(\Longleftrightarrow \lambda$ is a root of unity), then $z_{0}$ is a parabolic point. It is most of times non-linearizable. If $\theta \notin \mathbb{Q}$, then $z_{0}$ is called an irrationally indifferent periodic point. Several subcases occur. In the linearizable case, the Siegel disk refers to the
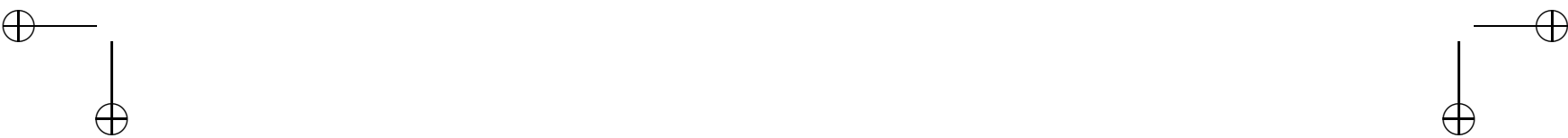
maximal domain on which a conjugacy to a rotation on a disk is possible. In the non-linearizable case, $z_{0}$ is called a Cremer point, and the dynamics becomes very complicated.

The basin of an attracting cycle is the set of points whose orbit tends to the cycle (i.e. end up staying in every neighborhood of the cycle). The filled-in Julia set $K(P)$ of a polynomial $P$ of degree $\geq 2$ is the complement of the basin of $\infty$. It is a non-empty compact subset of $\mathbb{C}$. The Julia set $J(P)$ has three equivalent and standard definitions. It is the boundary of $K(P)$ (which is the same as the boundary of the basin of $\infty$ ). It is the closure of the set of repelling periodic points. It is the complement of the Fatou set $\mathcal{F}$, where $z \in \mathcal{F} \Longleftrightarrow$ there exists a neighborhood $V$ of $z$ on which the family $\left\{P^{n}\right\}_{n \in \mathbb{N}}$ is normal.

A degree 2 polynomial has a connected Julia set $J(P) \Longleftrightarrow K(P)$ is connected $\Longleftrightarrow$ the (unique) critical point of $P$ belongs to $K(P)$. The Mandelbrot set $M$ is the set of $c \in \mathbb{C}$ such that the quadratic polynomial $P_{c}(z)=z^{2}+c$ has a connected Julia set. It is thus also the set of $c$ such that the critical point $z=0$ of $P_{c}$ has a bounded orbit. The latter definition is used for making computer pictures of $M$.

A hyperbolic polynomial $P$ is one which has an iterate $P^{n}, n>0$, whose derivative is expanding (for the spherical metric) on all its Julia set.

\section{Status of the hunt at the time of the conference}

This section chiefly presents the content of the talk I gave for Hamal's $60^{\text {th }}$ birthday conference, in a more detailed way and completed by references.

\subsection{Motivation}

The Julia set is the locus of chaos. To have a Julia set of positive measure means that a randomly chosen point in the Riemann sphere has a non zero probability of being in the locus of chaos. It is interesting in itself to know whether or not this may happen for polynomials.

Yet, the question has strong links with a set of still open conjectures:

Conjecture (Fatou) In the set of degree 2 polynomials, hyperbolic ones are dense. $^{2}$

Conjecture (MLC) The Mandelbrot set is locally connected.

Conjecture (NILF) The Julia set of a quadratic polynomial carries no invariant line field. ${ }^{3}$

\footnotetext{
${ }^{2}$ Fatou also conjectured that this holds in the set of degree $d$ polynomials and in the set of degree $d$ rational maps.

${ }^{3}$ See $[\mathrm{Mc}]$ for the definitions of a line field and the invariance thereof.
} 
Let us also label this article's main question:

Conjecture (Zero) The Lebesgue measure of Julia sets of quadratic polynomials is always equal to 0.

It was also conjectured for higher degree polynomials, and also for rational maps whose Julia set is not the whole Riemann sphere (there exist rational maps whose Julia set is the whole Riemann sphere).

We have the following known relations between them (as of 2005):

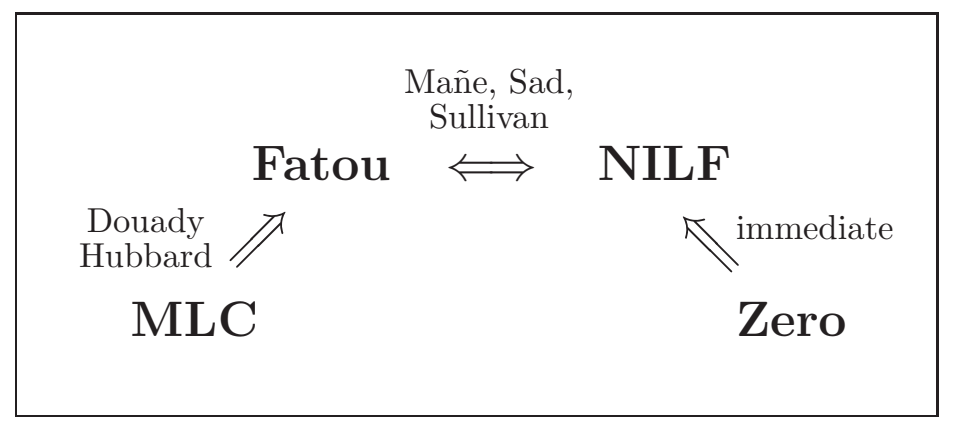

For $\mathrm{MLC} \Longrightarrow$ Fatou, see $[\mathrm{DH}]$ and $[\mathrm{Sc}]$.

For Fatou $\Longleftrightarrow$ NILF, see $[\mathrm{MSS}]$ and $[\mathrm{Mc}]$.

The Zero conjecture thus fitted well in the picture, all the more so as its analog for Kleinian group, Ahlfors' conjecture, was proved in 2004.

Yet, the Zero conjecture fails [BC].

\subsection{Known results (where not to look at) for quadratic polyno- mials}

In the rest of this article $P$ denotes a polynomial of degree $\geq 2$ (and most of times $=2), J(P)$ its Julia set, Leb the Lebesgue measure, $\operatorname{dim}_{\mathrm{H}}$ the Hausdorff dimension, $\mathrm{M}$ the Mandelbrot set, and $\mathrm{Pol}_{2}$ the set of polynomials of degree 2, also called quadratic polynomials. All degree 2 polynomials are $\mathbb{C}$-affine conjugated to a unique polynomial of the form $Q_{c}=z^{2}+c$.

Recall that a hyperbolic polynomial or rational fraction is one which has an iterate whose derivative is expanding (for the spherical metric) on all its Julia set. This is equivalent to all the critical points belonging to attracting or superattracting basins. The Lebesgue measure of such a Julia set is then equal to 0 (see $[\mathrm{DH}])$, and even better (see $[\mathrm{Su}])$ :

Theorem 1.1 (Sullivan) A hyperbolic rational fraction has a Julia set $J$ of Hausdorff dimension $<2$ (thus zero Lebesgue measure):

$$
\operatorname{dim}_{\mathrm{H}} J<2 \text {. }
$$
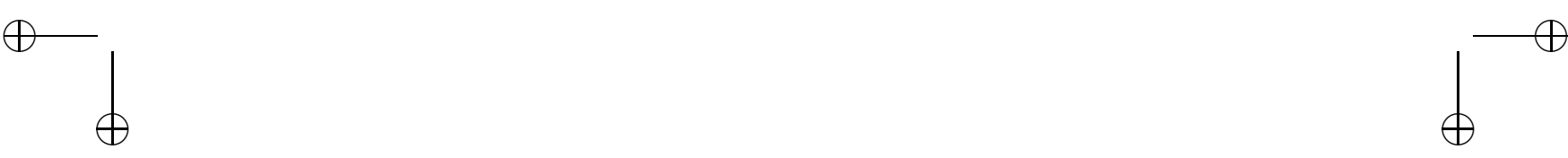
Let's recall a basic fact (yet not obvious):

Theorem 1.2 (Fatou, Julia, Douady, Hubbard) A degree 2 polynomial has at most one non repelling cycle.

The proof of this theorem can be found in [D2] and [B1]. Next, by $[\mathrm{L}]$ :

Theorem 1.3 (Lyubich, Shishikura) If $P$ has no indifferent periodic points and is not infinitely renormalizable, then

$$
\text { Leb } J(P)=0 .
$$

So, to find positive measure in $\mathrm{Pol}_{2}$, we must look either

- at infinitely renormalizable polynomials

- at polynomials with an indifferent cycle

These two conditions are mutually exclusive, and prevent $P$ from being hyperbolic.

The three theorems mentioned above show that there are plenty of "small" Julia sets, with different notions of what is "small". Let us cite a theorem by $[\mathrm{Sh}]$ providing plenty, in some sense, of Julia sets that are "big", in some sense:

\section{Theorem 1.4 (Shishikura)}

(S1) For a Baire generic set of values of $c \in \partial \mathrm{M}, P=z^{2}+c$ has a Hausdorff dimension 2 Julia set.

(S2) For a Baire generic set of values of $\theta \in \mathbb{R}, P=e^{2 i \pi \theta} z+z^{2}$ has a Hausdorff dimension 2 Julia set.

Remark 1.5 This may sound like an encouragement, but in case (S1), for a Baire generic set of values of $c \in \partial \mathrm{M}, P$ has no indifferent cycle, and is not renormalizable, thus by a previously mentioned theorem: Leb $J(P)=0$.

Let us focus on quadratic polynomials with an indifferent cycle. According to the Douady-Hubbard theory of tuning (quadratic-like renormalization of quadratic maps, [DH2]) it is enough to study the case where the cycle has period 1 . Then, $P$ is $\mathbb{C}$-affine conjugated to

$$
P_{\theta}: z \mapsto e^{2 i \pi \theta} z+z^{2}
$$

for a unique $\theta \in \mathbb{R}$. In the rational case we have $[\mathrm{ADU}]$ :

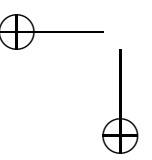




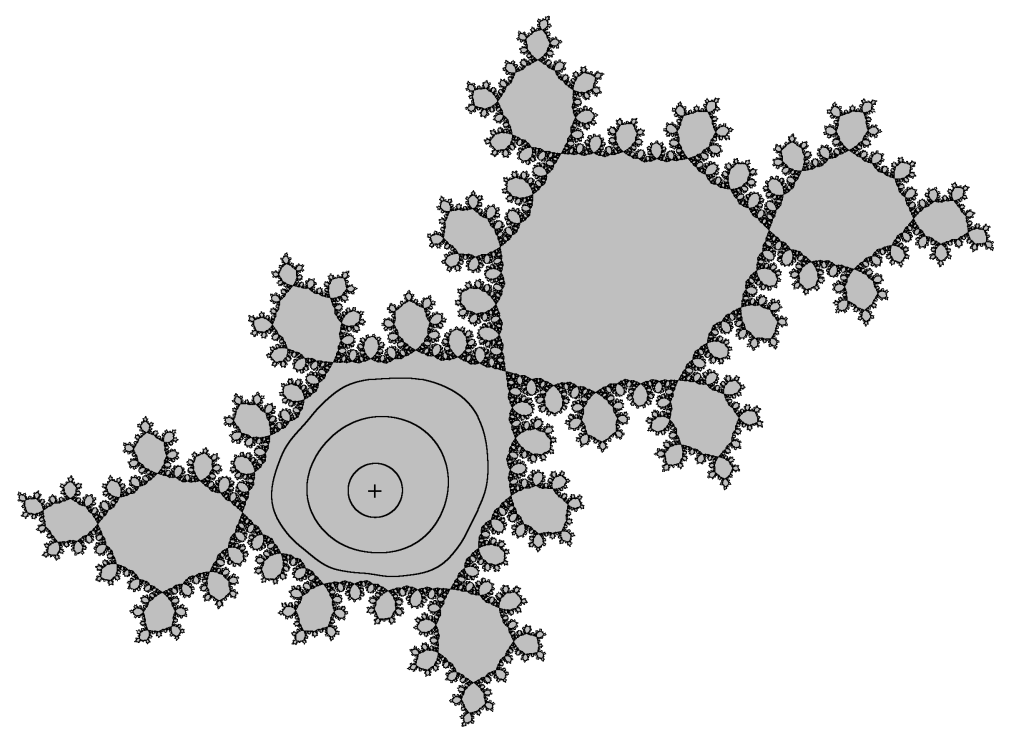

Figure 3.1. The Julia set of $P_{\theta}$ for $\theta=$ the golden mean $=\frac{1+\sqrt{5}}{2}$. The basin of infinity is in white, the Julia set in black and the rest in gray. The latter consists in the Siegel disk and its iterated preimages. We also drew a few invariant curves within the Siegel disk and marked its center $z=0$ with a cross.

Theorem 1.6 (Aaronson, Denker, Urbanski) If $\theta \in \mathbb{Q}$ then

$$
\operatorname{dim}_{\mathrm{H}} J\left(P_{\theta}\right)<2 \text {. }
$$

Recall that the set of bounded type irrationals, i.e. of numbers $\theta=$ $a_{0}+\frac{1}{a_{1}+\ddots}$ with $a_{n}$ bounded, has measure equal to 0 . From [Mc2]:

Theorem 1.7 (McMullen) If $\theta$ is a bounded type irrational then

$$
\operatorname{dim}_{\mathrm{H}} J\left(P_{\theta}\right)<2 .
$$

Let PZ be the set of irrational $\theta=a_{0}+\frac{1}{a_{1}+\ddots}$ such that

$$
\ln a_{n}=\mathcal{O}(\sqrt{n})
$$

This is a set of full measure in $\mathbb{R}$. By $[\mathrm{PZ}]$ :
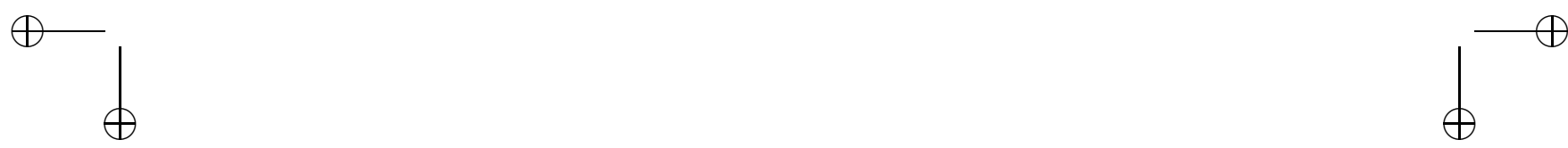
Theorem 1.8 (Petersen, Zakeri) If $\theta \in \mathrm{PZ}$, then $z=0$ is linearizable and

$$
\text { Leb } J(P)=0 \text {. }
$$

\subsection{Douady's plan}

It takes place in the family

$$
P_{\theta}(z)=e^{2 i \pi \theta} z+z^{2}
$$

with $\theta \in \mathbb{R}$. We recall that $z=0$ is an indifferent fixed point and that all other periodic points are repelling (by Theorem 1.2).

Conjecture (Douady) There exists a value of $\theta \in \mathbb{R}$ such that $z=0$ is a Cremer point of $P_{\theta}$ and such that

$$
\text { Leb } J\left(P_{\theta}\right)>0 \text {. }
$$

In short, the idea is to look at the filled-in Julia set $K\left(P_{\theta}\right)$ when its interior is not empty and perturb $\theta$ so as to lose much interior but not too much measure. Let us detail this.

Let us list a few properties concerning $J=J(P)$ and $K=K(P)$ where $P$ is a polynomial of degree at least 2 :

- (Fatou, Julia) $J=\partial K$,

- (Fatou, Julia, Sullivan) $\operatorname{int}(K) \neq \varnothing \Longleftrightarrow K$ has a cycle which is either attracting, parabolic or Siegel,

- (Douady [D]) the map $P \mapsto \operatorname{Leb}(K)$ is upper semi-continuous.

The idea is then to construct a sequence of $\theta_{n} \in \mathbb{R}$ such that

(D1) $\theta_{n} \longrightarrow \theta \in \mathbb{R}$

(D2) each $P_{\theta_{n}}$ is Siegel or parabolic at 0 (then int $\left.K\left(P_{\theta_{n}}\right) \neq \varnothing\right)$,

(D3) $P_{\theta}$ is Cremer at $z=0$,

(D4) Lebint $\left(K\left(P_{\theta_{n}}\right)\right)$ is bounded below,

i.e. we do not lose too much measure between two successive $\theta_{n}$. Then, by (D1), (D4) and the semi-continuity mentioned above, Leb $K\left(P_{\theta}\right)>0$. By (D3), $\operatorname{int}\left(K\left(P_{\theta}\right)\right)=\varnothing$, i.e. $J\left(P_{\theta}\right)=K\left(P_{\theta}\right)$ :

$$
\text { Leb } J\left(P_{\theta}\right)>0 \text {. }
$$

To build such a sequence, $\theta_{n+1}$ is defined as a perturbation of $\theta_{n}$. The main problem is to ensure that the measure loss for the interior of the filled-in Julia set is small, so as to have (D4) hold. Adrien proposed to do this in two steps:

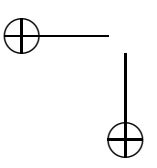


- Start from a $\theta_{n} \in \mathbb{R} \backslash \mathbb{Q}$ with $P_{\theta_{n}}$ having a Siegel disk, and take $\theta_{n+1} \in \mathbb{Q}$ to be one of its convergents.

- Start from $\theta_{n+1} \in \mathbb{Q}$ and take $\theta_{n+2} \in \mathbb{R} \backslash \mathbb{Q}$ close to $\theta_{n+1}$ to be one of its special irrational perturbations, as described below. Such a $P_{\theta_{n+2}}$ has a Siegel disk.

It is easy to get (D3) if the perturbations can be taken arbitrarily small. The special sequence of irrational perturbations $\left(\alpha_{k}\right)_{k \in \mathbb{N}}$ of a rational $p / q$ is defined as follows: write one of the two (necessarily finite) continued fraction expansions

$$
p / q=\left[a_{0}, \ldots, a_{m}\right]=a_{0}+\frac{1}{a_{1}+\frac{1}{\ddots \cdot+\frac{1}{a_{m}}}}
$$

then $\operatorname{let}^{4}$

$$
\alpha_{k}=\left[a_{0}, a_{1}, \ldots, a_{m}, k, 1,1, \ldots\right] .
$$

So here is a Wish list he asked me to check as a $\mathrm{PhD}$ thesis subject:

Wish 1 Let $\alpha$ be such that $P_{\alpha}$ has a Siegel disk (with possible additional conditions, like " $\alpha$ has bounded type"). Let $p_{n} / q_{n}$ be the continued fraction convergents of $\alpha$. Then

$$
\liminf _{n \rightarrow+\infty} \operatorname{Leb} \stackrel{\circ}{K}\left(P_{p_{n} / q_{n}}\right) \geq \operatorname{Leb} \stackrel{\circ}{K}\left(P_{\alpha}\right),
$$

in other words, the loss of measure of interiors is tame.

This allows to do Adrien's first step: replacing some $\theta_{n}$ for which $P_{\theta_{n}}$ has a Siegel disk by one of its convergent without losing too much measure, and calling this convergent $\theta_{n+1}$.

Yet, when one perturbs a rational, the theory of parabolic implosion [D] easily implies that there is a definite loss of measure, which necessarily complicates the second step:

$$
\limsup _{\alpha \longrightarrow p / q} \operatorname{Leb} K\left(P_{\alpha}\right)<\operatorname{Leb} K\left(P_{p / q}\right)=\operatorname{Leb} \stackrel{\circ}{K}\left(P_{p / q}\right) .
$$

To miminize this loss, Adrien proposed to take the special sequence $\alpha_{k} \longrightarrow$ $p / q$ described above.

\footnotetext{
${ }^{4}$ It is chosen so that in the cylinder renormalization associated to the parabolic implosion at $\theta_{n+1}$, the virtual multiplier $=$ the golden mean.
} 
Let $\operatorname{loss}(p / q)$ be defined by:

$$
\frac{\liminf _{k \rightarrow+\infty} \operatorname{Leb} \stackrel{\circ}{K}\left(P_{\alpha_{k}}\right)}{\operatorname{Leb} K\left(P_{p / q}\right)}=1-\operatorname{loss}(p / q),
$$

which is sort of a measure of the area loss for this special sequence. One has $0<\operatorname{loss}(p / q)<1$ (the lower bound follows from the definite loss aforementioned, the upper bound from the fact that the Siegel disk of cannot shrink too much in terms of its area, which is proved using the the theory of parabolic implosion and a lower bound on the size of Siegel disks with a fixed rotation numer (diophantine in our case), due to Siegel. See also my thesis $[\mathrm{C}]$ completed with [C2]).

Wish 2 With possible additional conditions, for big values of $q, \operatorname{loss}(p / q)$ is arbitrarily close to 0 .

Then in the second step of Adrien's plan (defining the irrational $\theta_{n+2}$ once the rational $\theta_{n+1}$ has been fixed), to keep the loss of measure small so that (D4) can hold, it is necessary that in the previous step (deciding which approximant of the irrational $\theta_{n}$ the rational $\theta_{n+1}$ shall be), we took the approximant deep enough, so that its denominator is big, so that the quantity $\operatorname{loss}\left(\theta_{n+1}\right)$ is small.

\subsection{Analysis of the plan, reduction}

Based on the preparatory work of Jellouli [J], I gave a positive answer to Wish 1 in my thesis [C]:

Theorem 1.9 (Chéritat) Wish 1 is granted, without any additional condition on $\alpha$.

To prove this theorem, it was enough to prove that the set $U_{n}$ of points which under iteration of $P_{p_{n} / q_{n}}$ never leave the Siegel disk $\Delta$ of the unperturbed map, takes most of the area of $\Delta$, in the sense that $\operatorname{Leb}\left(\Delta \backslash U_{n}\right) \longrightarrow 0$ as $n \longrightarrow+\infty$. This is done by comparing the dynamics to a vector field. For the expert reader, let us mention that the comparison is made possible thanks to a control on parabolic explosion, allowed by Yoccoz's inequality on the size of the limbs of the Mandelbrot set. See [C] for details.

Towards Wish 2, I gave in my thesis an upper bound on $\operatorname{loss}(p / q)$ in terms of the image of the basin of $\infty$ in Fatou coordinates (see Figure 3.5 for an illustration): let us put the natural Euclidean metrics on $\mathbb{C} / \mathbb{Z}$ inherited from $\mathbb{C}$, and do the same with the Lebesgue measure. For $p / q \in \mathbb{Q}$, the basin of $\infty$ for $P_{p / q}$ is an open set, and its image in a repelling cylinder of the parabolic point is a bounded non empty open set $U=U(p / q)$. Let
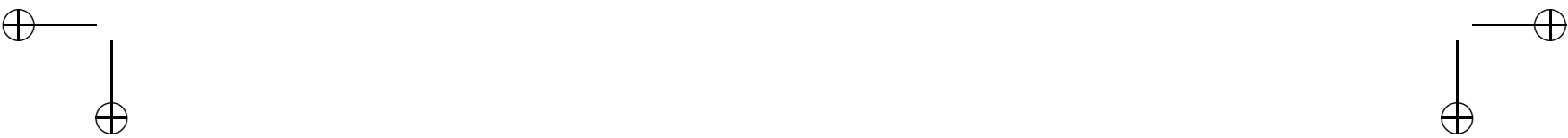


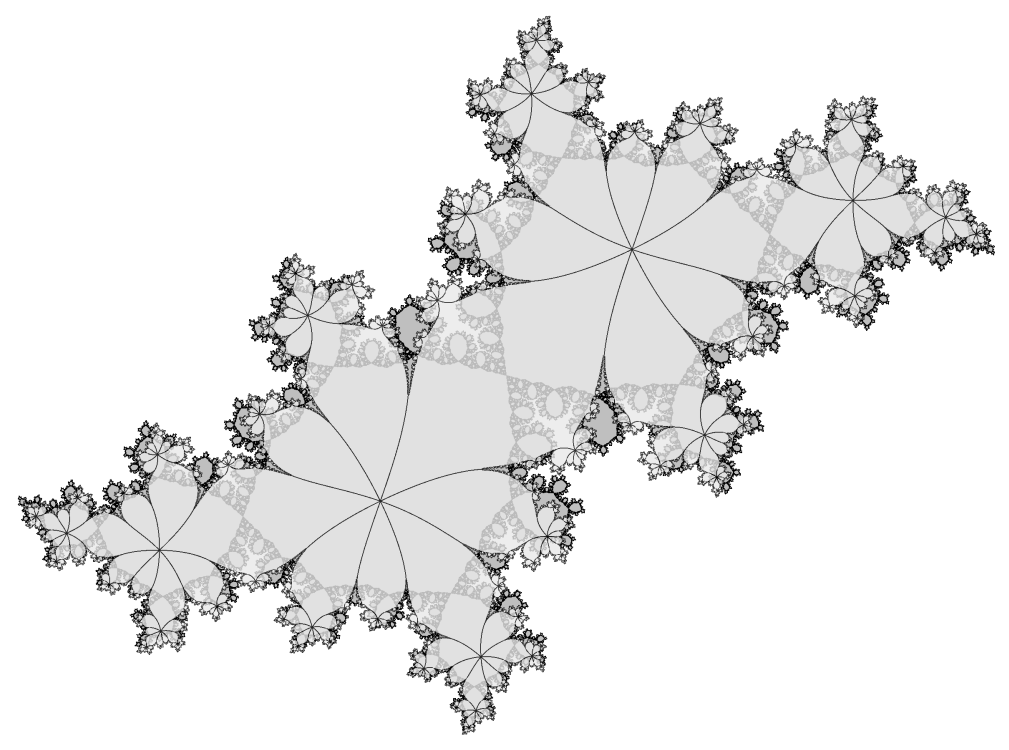

Figure 3.2. On this picture, we superposed (semi-transparent) the Julia set of $P_{\alpha}$ for the approximant $\alpha=13 / 8$ of the golden mean over the Julia set for $\alpha=$ the golden mean. Adrien's Wish 1 was based on that kind of pictures. In particular, notice how thin is the intersection between the unperturbed Siegel disk and the basin of infinity for the perturbed Julia set.

- $A(p / q)$ be its Lebesgue measure, and

- $h(p / q)$ its height: $h(p / q)=\sup _{z \in U} \operatorname{Im} z-\inf _{z \in U} \operatorname{Im} z$.

The Figure 3.5 shows an example.

Theorem 1.10 (Chéritat) There exists a function $m\left(A^{\prime}, h^{\prime}\right)$ of $A^{\prime}>0$ and $h^{\prime}>0$ s.t.

1. $\forall p / q \in \mathbb{Q}$, if $A(p / q) \leq A^{\prime}$ and $h(p / q) \leq h^{\prime}$ then

$$
\operatorname{loss}(p / q) \leq m\left(A^{\prime}, h^{\prime}\right)
$$

2. for all fixed $h^{\prime}>0, m\left(A^{\prime}, h^{\prime}\right) \longrightarrow 0$ as $A^{\prime} \longrightarrow 0$.

See [C]. Hence if $U(p / q)$ has small area and not too big height, then the loss is small, allowing Adrien's plan to work: 

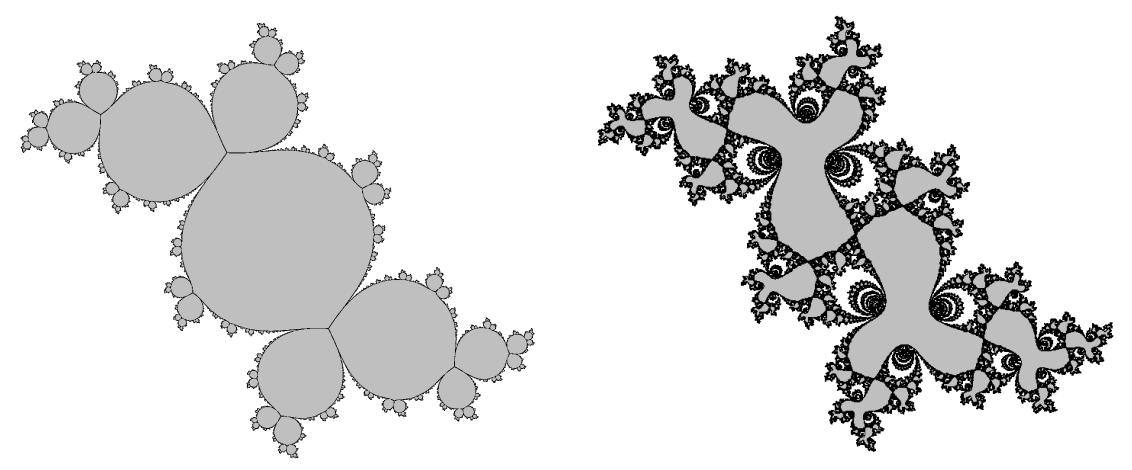

Figure 3.3. The fat Douady rabbit $(\alpha=1 / 3=[0,3]=[0,2,1])$, and one of its perturbations $\left(\alpha=\left[0,2,1, k, \frac{1+\sqrt{5}}{2}\right]\right.$, for $\left.k=150\right)$.

Corollary 1.11 If the next conjecture holds, then $\exists \theta$ such that Leb $J\left(P_{\theta}\right)>0$.

To state this conjecture, let us say that an irrational $\alpha$ is gilded if its continued fraction expansion has its entries eventually all equal to 1: $\alpha=$ $\left[a_{0}, \ldots, a_{k}, 1,1, \ldots\right]$.

Conjecture (Chéritat) For all gilded irrational $\alpha$, denoting $p_{n} / q_{n}$ its convergents, we have

(C1) $A\left(p_{n} / q_{n}\right) \longrightarrow 0$

(C2) $h\left(p_{n} / q_{n}\right)$ is bounded.

Backing this conjecture, Figure 3.6 is the kind of experiment that allowed me to convince Adrien that his method worked.

Proof (of Corollary 1.11): The above conjecture with Theorem 1.10 imply that for all gilded irrational $\alpha, \operatorname{loss}\left(p_{n} / q_{n}\right) \longrightarrow 0$ where $p_{n} / q_{n}$ denote the convergents of $\alpha$. Now we want to construct a sequence $\theta_{n}$ satisfying conditions (D1) to (D4) of Douady's plan. Let $\theta_{0}$ be any gilded irrational, for instance the golden mean. Let $\theta_{n}$ be defined by induction according to the two steps of Douady's plan, which are reminded here: $\theta_{2 p}$ is irrational, $\theta_{2 p+1}$ is one of its convergents and is thus rational, $\theta_{2 p+2}$ is a special gilded irrational perturbation of the rational number $\theta_{2 p+1}$. We claim we can choose the sequence $\left(\theta_{n}\right)$ so that for all $n>0$ :

$$
\text { Leb } \stackrel{\circ}{K}\left(P_{\theta_{n}}\right)>\left(1-2^{-n}\right) \text { Leb } \stackrel{\circ}{K}\left(P_{\theta_{n-1}}\right) \text {. }
$$
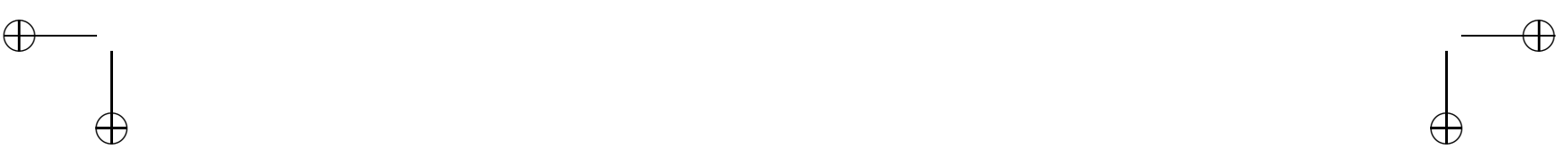


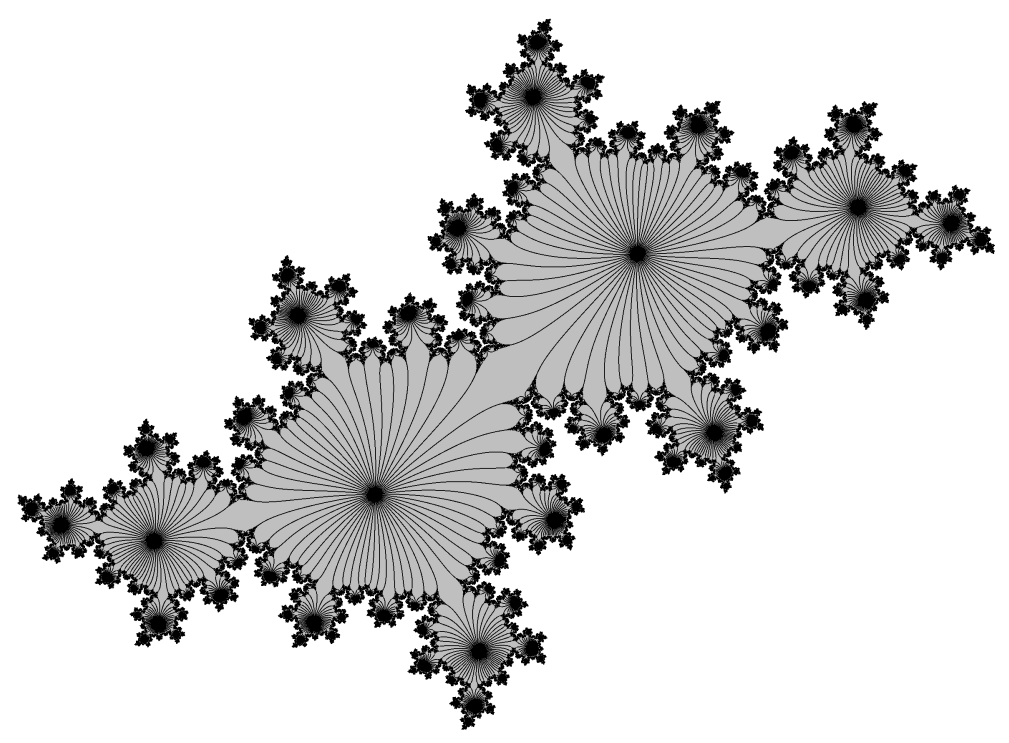

Figure 3.4. The Julia set for $\alpha=89 / 55$ (9-th approximant to the golden mean). Compare with Figure 3.1.

Given $\theta_{2 p}$, the existence of a convergent $\theta_{2 p+1}$ satisfying (3.1) with $n=$ $2 p+1$ is granted by Theorem 1.9. We moreover choose $\theta_{2 p+1}$ so that $\operatorname{loss}\left(\theta_{2 p+1}\right)<2^{-2 p-2}$. Therefore, there exists a special perturbation $\theta_{2 p+2}$ of $\theta_{2 p+1}$ so that (3.1) holds for $n=2 p+2$. The sequence $\left(\theta_{n}\right)_{n \in \mathbb{N}}$ thus satisfies (D2) and (D4). To have it also satisfy (D1) it is enough to require at each step that $\left|\theta_{n+1}-\theta_{n}\right|<2^{-n}$, which is possible since in the construction above, there is a whole sequence of valid choices of $\theta_{n+1}$, sequence which tends to $\theta_{n}$. So now the sequence $\theta_{n}$ tends to some $\theta$. To also satisfy (D3), it is enough to have a $\theta$ that satisfies Cremer's Liouvillian sufficient condition (see $[\mathrm{M}]$ ) for non-linearizability, which can be achieved for instance by requiring that $\max \left(\left|\theta_{2 p+1}-\theta_{2 p}\right|,\left|\theta_{2 p}-\theta_{2 p-1}\right|\right)<1 / \exp \left(p \cdot 2^{q}\right)$ where $q$ is the denominator of $\theta_{2 p-1}$.

\subsection{Analogy with McMullen's results}

This subsection is rather informal, because first the details are a bit complicated, and second at that time we did not know how to make it work. The results we are referring to will be found in [Mc2].

McMullen proved that the critical point is a Lebesgue density point 

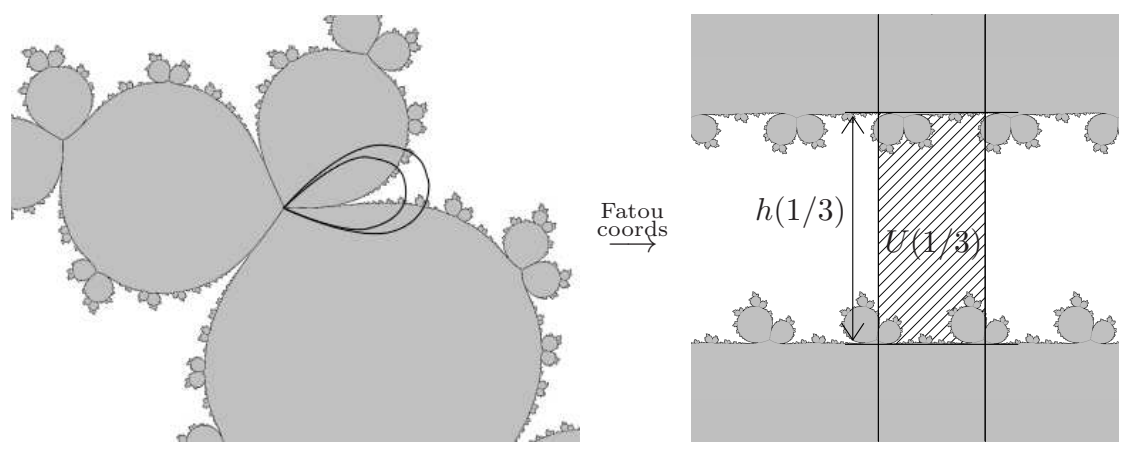

Figure 3.5. Left: closeup at the parabolic fixed point on the Julia set of $P_{\alpha}$ for $\alpha=1 / 3$. We sketched a fundamental domain. By $P_{1 / 3}^{3}$, its inner boundary is mapped to the outer, and the quotient is a Riemann surface isomorphic to $\mathbb{C} / \mathbb{Z}$, called the Ecalle-Voronin cylinder. The isomorphism is called the Fatou coordinate. Right: image of the Julia set by the Fatou coordinate. The cylinder $\mathbb{C} / \mathbb{Z}$ has been unwrapped: the picture is invariant by $z \mapsto z+1$ and vertical lines mark a fundamental domain of the cylinder $\mathbb{C} / \mathbb{Z}$. We hatched its intersection with the basin of $\infty$. The quantities $h$ and $A$ are the euclidean height and area of the hatched set.

of the interior of the filled-in Julia set of $P_{\alpha}$ for any bounded type $\alpha$. Now, the pictures of the parabolic filled-in Julia sets $K\left(P_{p_{n} / q_{n}}\right)$ in Fatou coordinates bear some kind of resemblance to that of zooms on the critical point of the Siegel disk bearing filled-in Julia set $K\left(P_{\alpha}\right)$. This is illustrated in Figure 3.7.

Could McMullen's density results be adapted to our setting, i.e. to the parabolic filled-in Julia sets seen in Fatou coordinates, and yield my conjecture, henceforth positive measure?

McMullen proved more: for instance, when the entries of the continued fraction of $\alpha$ are eventually periodic of period $p$, then there exist a map $\lambda: z \mapsto \tau z$ if $p$ is even and $\tau \bar{z}$ if $p$ is odd, with $\tau \in \mathbb{C}, 0<|\tau|<1$ and analytic functions $g_{k}$ such that the sequence

$$
\lambda^{-n} \circ P_{\alpha}^{q_{n+k p}} \circ \lambda^{n} \underset{n \rightarrow+\infty}{\longrightarrow} g_{k} .
$$

We conjecture ${ }^{5}$ the existence of a similar tower of limits $\widetilde{g}_{k}$

$$
\lambda^{-n} \circ P_{p_{n+k p} / q_{n+k p}}^{q_{n+k p}} \circ \lambda^{n} \underset{n \rightarrow+\infty}{\longrightarrow} \widetilde{g}_{k} .
$$

Figure 3.8 shows closeups on the Julia sets for the convergents $p_{n} / q_{n}$ of the golden mean (whose period $p=1$ ), with $n$ ranging from 5 to 13 . In

\footnotetext{
${ }^{5}$ This conjecture is still unproven today. We found another way to transfer McMullen's Lebesgue density result to parabolic filled-in Julia sets seen in cylinders.
} 


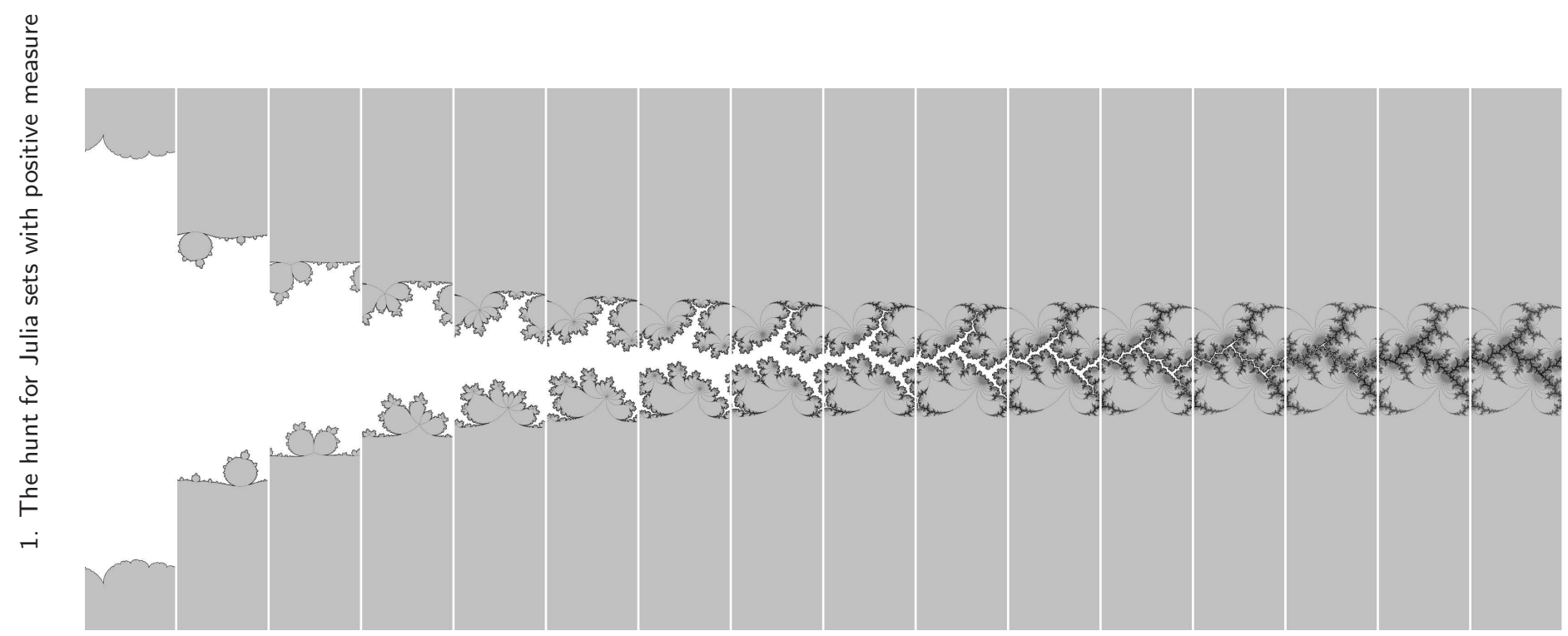

Figure 3.6. The interior of the filled-in Julia set of $P_{p_{n} / q_{n}}$ (gray), the Julia set (black), and basin of infinity (white), seen in Fatou coordinates, for the first 16 approximants $p_{n} / q_{n}$ of the golden mean. Each time we drew a fundamental domain for $\mathbb{C} / \mathbb{Z}$, using the same scale, and flipped a strip upside down every two strip, so that a convergence is clearly visible. Of course we put only a finite part of each infinite strip but what is missed is just plain grayness. It really looks like the white set has bounded height and measure tending to 0 . But keep in mind that the black set is in the closure of the white, so that some measure may hide there even if it looks unlikely (for instance the fjords are extremely thin). 
each frame, the scale has been set so that the distance between the critical point (at the center of the frame) and its $q_{n}$-th iterate, is constant (about a 16th of the frame width). The convergence of these Julia sets echoes the aforementioned conjectured convergence of the rescaled $q_{n}$-th iterate.

\subsection{What remained to be done}

Together with Xavier Buff, we were addressing my conjecture (on the areas $A\left(p_{n} / q_{n}\right)$, and heights $\left.h\left(p_{n} / q_{n}\right)\right)$ via the study of cylinder renormalization (Buff, Epstein, Shishikura, Yampolsky, ... ). ${ }^{6}$ Let us denote $\mathcal{R}$ this renormalization.

H. Inou and M. Shishikura had recently proved the existence of a stable class for $\mathcal{R}$. This was, and still is, done by perturbation of the parabolic case thus requires the rotation number to be close enough to 0 . In particular, there is an integer $N>0$ such that this class contains the quadratic polynomials $P_{\alpha}$ for which $\alpha$ has all its continued fraction entries $\geq N$.

In the discussion above, we used gilded numbers, i.e. numbers whose continued fraction entries are eventually constant equal to 1 . The whole discussion is valid if instead we look at numbers whose continued fraction entries are eventually constant equal to $N$. With this modification, Inou and Shishikura's result proved part $(\mathrm{C} 2)$ of my conjecture (i.e. the height $h\left(p_{n} / q_{n}\right)$ is bounded).

We then had a framework to attack part (C1) (which claims that the area $A\left(p_{n} / q_{n}\right)$ should tend to 0$)$.

\section{After the conference}

\subsection{Present results}

Xavier Buff and I finished the proof during the summer following the conference (2005). To bound the area $A\left(p_{n} / q_{n}\right)$, we first used Inou-Shishikura's results to prove that the post-critical set of $P_{p_{n} / q_{n}}$ stays arbitrarily close to the Siegel disk $\Delta$ of $P_{\alpha}$ :

\footnotetext{
${ }^{6}$ Roughly, it consists in the following: draw a well chosen curve $C$ between the fixed point 0 and the other fixed point of $P_{\alpha}$. Among other conditions, we want that $P_{\alpha}$ be injective on $C$ and send it to a curve disjoint from $C$ apart from its endpoints. Consider the bounded region $D$ bounded by $C$ and $P_{\alpha}(C)$. Consider the first return map from $D$ to $D$. Now glue the boundary of $D$ by identifying $z \in C$ with $P_{\alpha}(z)$. Remove the two endpoints from this. One gets a Riemann surface isomorphic to a cylinder, or if one prefers to $\mathbb{C}^{*}$. The first return map is conjugated by this isomorphism to a map which is the cylinder renormalization of $P_{\alpha}$. The fixed point 0 of $P_{\alpha}$ corresponds to one end of the cylinder, and drawing another curve from this end to a fixed point of the first return map allows to renormalize the renormalized map, and so on...
} 

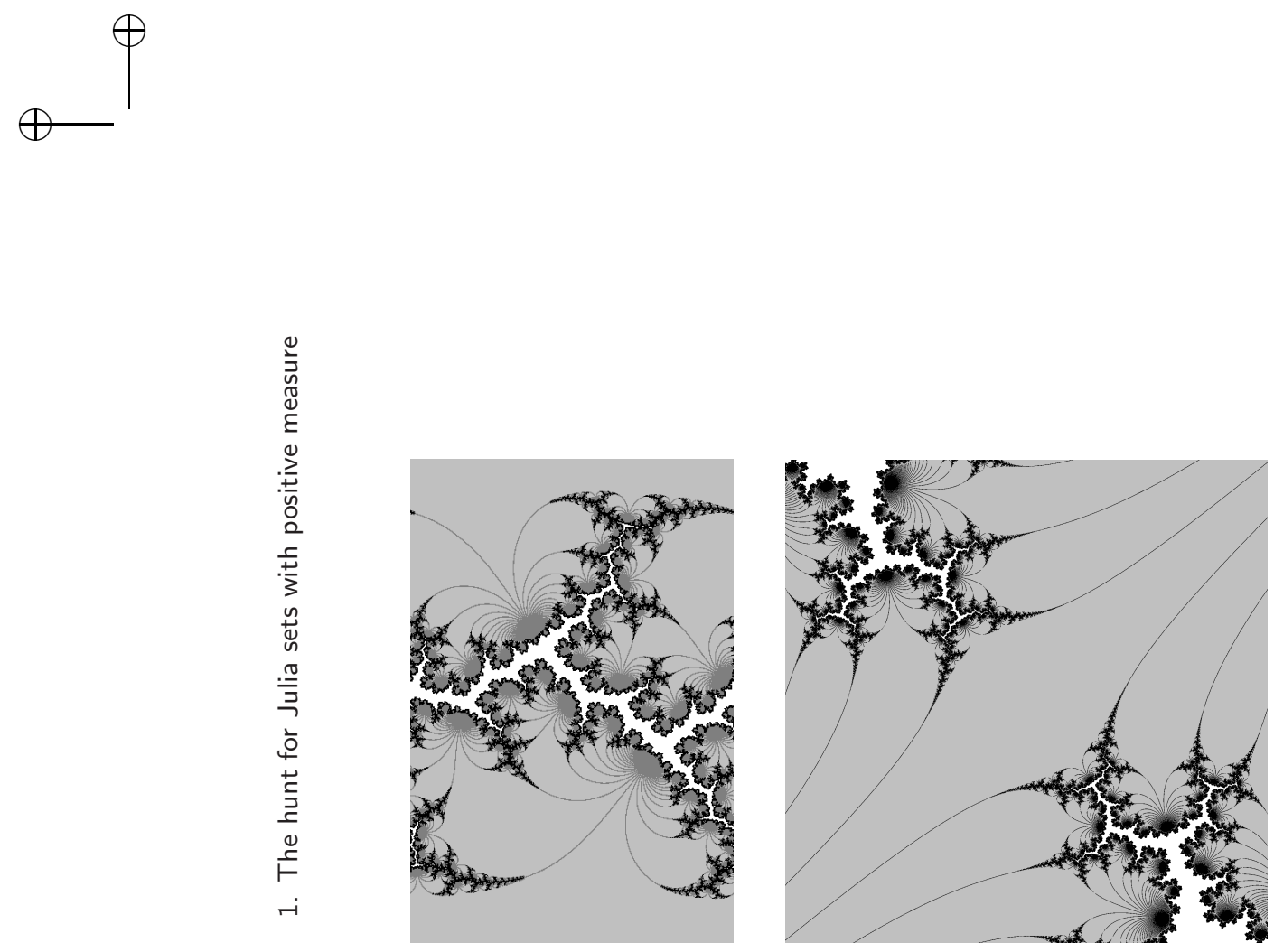

$$
\frac{233}{144}
$$

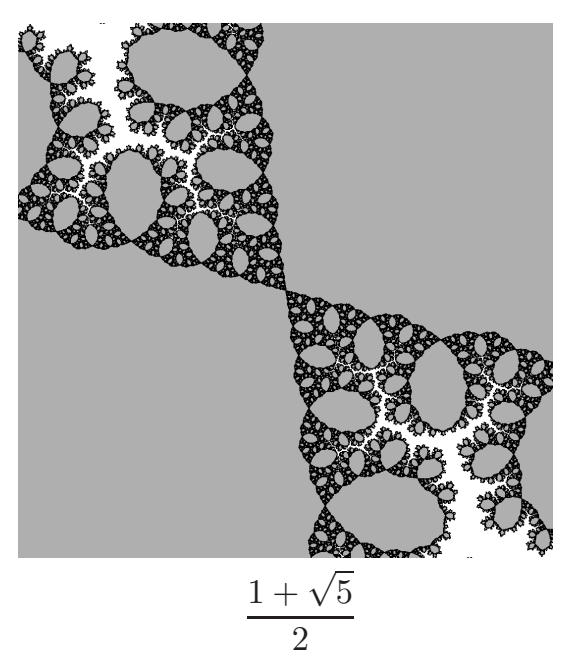

Figure 3.7. The image on the left shows the Julia set of $P_{p_{n} / q_{n}}$ in Fatou coordinate for some approximant $p_{n} / q_{n}$ of the golden mean. The image in the middle shows a closeup on the same Julia set, but in the initial coordinates, centered on the critical point. The image on the right shows a closeup at the same scale, also centered on the critical point, on the Julia set of $P_{\alpha}$ for $\alpha=$ the golden mean. There is some kind of resemblance, especially when one looks at the thick part of the basin of infinity. 
$\bigoplus$

4. After the conference
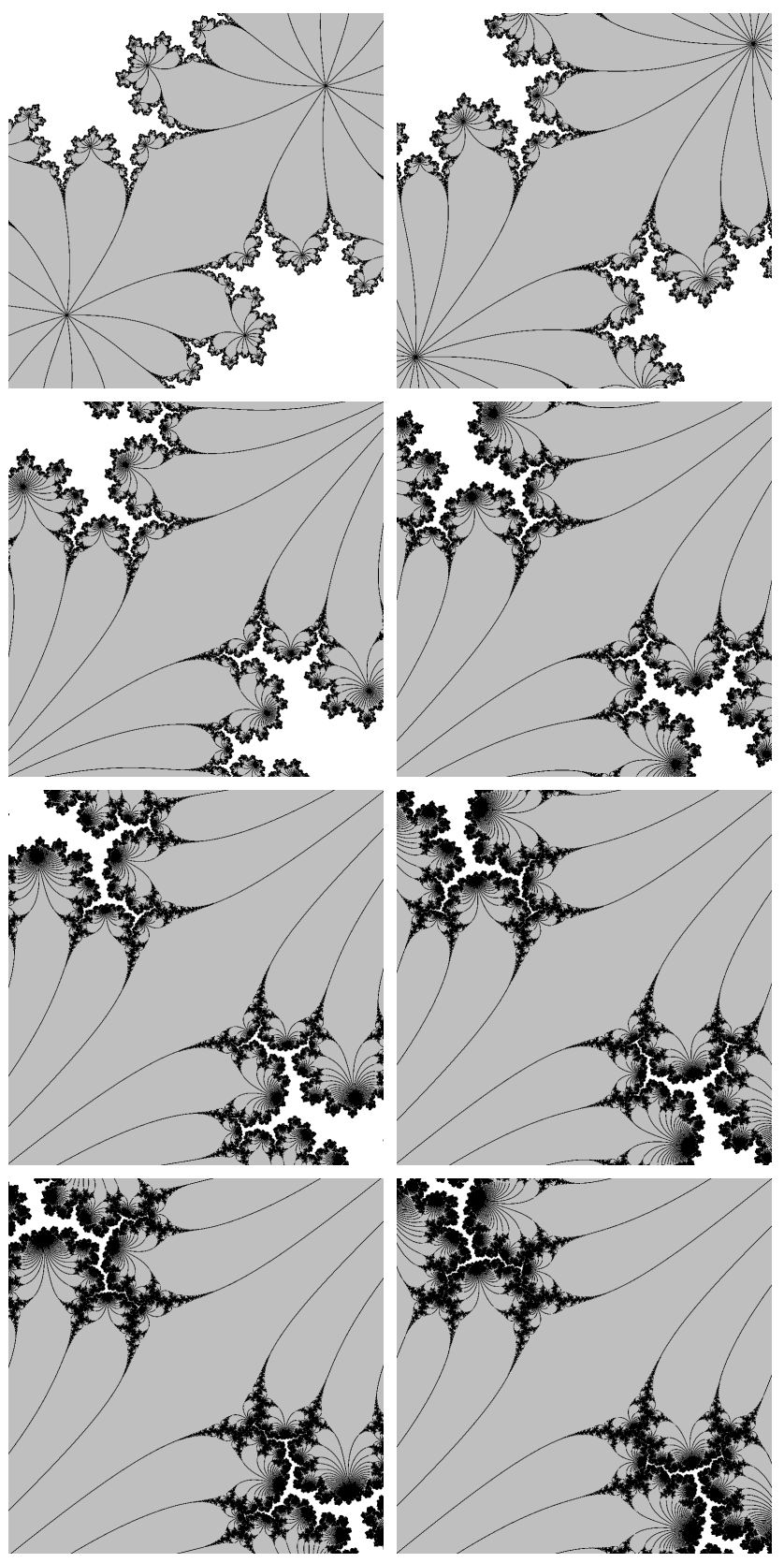

Figure 3.8. 
Theorem 1.12 Let $\alpha$ be any irrational with every continued fraction entry $\geq N$ and with moreover bounded type, i.e. the entries form a bounded sequence. Then $\forall \varepsilon>0, \exists n_{0}$ such that $\forall n \geq n_{0}$, every point in the postcritical set of $P_{p_{n} / q_{n}}$ is at distance $<\varepsilon$ from $\Delta$ (or in $\Delta$ ).

Then, using Wish 1 (which I proved earlier in my thesis), McMullen's theorem of Lebesgue density of int $K\left(P_{\alpha}\right)$ near $\Delta$, a pull-back argument, a lemma à la Vitali, and the bound on $h\left(p_{n} / q_{n}\right)$, it is easy to conclude and prove part $(\mathrm{C} 1)$ of my conjecture, thus finishing the proof of the existence of a Julia set with positive Lebesgue measure.

We also extended the method to yield examples of Julia sets with positive measure for quadratic polynomials with a Siegel disk, and also for infinitely renormalizable quadratic polynomials.

The preprint [IS] presenting the near-parabolic renormalization of Inou and Shishikura has been just been submitted; our preprint $[\mathrm{BC}]$ giving the full details of our positive measure result too.

\subsection{Prospects}

There are still open questions: ${ }^{7}$

- Does there exist a polynomial or a rational map, of degree $\geq 2$, with a Cremer point, but whose Julia set has Lebesgue measure $=0$ ?

- Quadratic Julia sets bearing a Cremer point, or a fixed Siegel disk not containing the critical point in its boundary, are non-locally connected. There is also a construction by Dan Sørensen of some infinitely renormalizable quadratic polynomials with a non-locally connected Julia set [So]. Could it happen that for quadratic polynomials, $J$ is non-locally connected $\Longleftrightarrow J$ has positive Lebesgue measure?

- Similarly, is it equivalent for a quadratic Julia set with a fixed Siegel disk $\Delta$, to have a positive measure Julia set and to have $\Delta$ not containing the critical point?

- Is it possible to characterize the set of irrational numbers $\alpha$ such that Leb $J\left(P_{\alpha}\right)>0$ ?

- Can we produce accurate computer pictures quadratic Julia sets with positive measure?

\footnotetext{
${ }^{7}$ Thanks to the people who formulated some of them.
} 
- Would one be able to see anything on an accurate picture? The problem being that around the critical point, the Julia set could be so dense that it would not be possible to see the difference between $J$ and a neighborhood of the Cremer point, unless one draws an astronomical number of pixels.

- Is $P$ ergodic on $J$ in our examples?

Moreover, let us mention the following open conjecture, which if it were true, would give another proof of the existence of Cremer point bearing quadratic Julia sets with positive measure:

\section{Conjecture (Hubbard)}

$$
\inf _{|\lambda|<1} \operatorname{Leb} K\left(\lambda z+z^{2}\right)>0
$$


$\bigoplus$ 


\section{Bibliography}

[ADU] J. Aaronson, M. Denker, M. Urbanski Ergodic Theory for Markov Fibered Systems and Parabolic Rational Map. Transactions of the AMS, Vol. 337, Nb. 2, pp. 495-548 (1993)

[Bl] P. Blanchard Complex Analytic Dynamics on the Riemann Sphere. Bulletin of the AMS, Vol. 11, Nb. 1, pp. 85-141 (1984)

[BC] X. Buff, A. ChÉRITAt Quadratic Julia Sets with Positive Area. arXiv:math/0605514

[C] A. ChÉRItAT Recherche d'ensembles de Julia de mesure de Lebesgue positive. Thesis, Université Paris Sud, Orsay (2001)

[C2] A. ChÉRITAT Semi-continuity of Siegel disks under parabolic implosion. arXiv:math/0507250

[D] A. Doundy Does a Julia set depend continuously on the polynomial? In Complex dynamical systems: The mathematics behind the Mandelbrot set and Julia sets. Ed. R.L. Devaney, Proc. of Symposia in Applied Math., Vol 49, Amer. Math. Soc., pp. 91-138 (1994)

[D2] A. DouAdy Systèmes dynamique holomorphes. Séminaire Bourbaki, 35e année, 1982/83, n 599. Astérisque 105-106, Société Mathématique de France (1983)

[DH] A. Douady, J.H. HubBard Étude dynamique des polynômes complexes. Publications mathématiques d'Orsay (1984-85)

[DH2] A. Douady, J.H. HubBard On the dynamics of polynomiallike mappings. Annales scientifiques de l'École Normale Supérieure Sér. 4, 18 no. 2, pp. 287-343 (1985)

[IS] H. Inou, M. Shishikura The renormalization for parabolic fixed points and their perturbation. Preprint 
[J] H. Jellouli Sur la densité intrinsèque pour la mesure de Lebesgue et quelques problèmes de dynamique holomorphe. Thesis, Université Paris Sud, Orsay (1994)

[L] M. LYUBICH On the Lebesgue measure of a quadratic polynomial. arXiv:math/9201285 / IMS at Stony Brook Preprint 1991/10 (1991)

[MSS] R. Mañe, P. Sad, D. Sullivan On the dynamics of rational maps. Annales scientifiques de l'École Normale Supérieure Sér. 4, 16 no. 2 , pp. $193-217$ (1983)

[Mc] C.T. McMullen Complex Dynamics and Renormalization. Annals of Mathematics Studies vol. 135 (1994)

[Mc2] C.T. MCMullen Self-similarity of Siegel disks and Hausdorff dimension of Julia sets. Acta Math. 180, no. 2, pp. 247-292 (1998)

[M] J. Milnor Dynamics in one complex variable, Introductory lectures, 2nd edition, Vieweg Verlag, Weisbaden, (2000)

[PZ] C. Petersen And S. Zakeri On the Julia set of a typical quadratic polynomial with a Siegel disk. Ann. of Math. (2) 159, no. 1, pp. 1-52 (2004)

[Sc] D. Schleicher On Fibers and Local Connectivity of Mandelbrot and Multibrot Sets. In Fractal Geometry and Applications: A Jubilee of Benoit Mandelbrot, Proceedings of Symposia in Pure Mathematics, AMS (2004)

[Sh] M. Shishikura The Hausdorff dimension of the boundary of the Mandelbrot set and Julia sets. Ann. of Math. (2) 147 , no. 2, pp. 225-267 (1998)

[So] D. SøRENSEN Infinitely renormalizable quadratic polynomials, with non-locally connected Julia set. J. Geom. Anal. 10, no. 1, pp. 169-206 (2000)

[Su] D. Sullivan Conformal dynamical systems, in Geometric dynamics. (Rio de Janeiro, 1981) Springer Verlag Lecture Notes Vol. 1007, pp. 725-752 (1983)

[T] The Mandelbrot Set, Theme and Variations, London Mathematical Society Lecture Note Series 274, Ed. Tan Lei. 
$\bigoplus$

Bibliography 